\title{
Clonidine versus Morphine as additives to Bupivacaine in patient-controlled epidural analgesia for postoperative pain after abdominal hysterectomy
}

\author{
Authors: Cindea I., Balcan A., Gherghina V., Samoila B., Buzatu G. \\ Emergency Clinical Hospital of Constanta - Department of Anaesthesia \& Intensive Care, Constanta, Romania
}

Background and goal of study:

We planned this study to compare in terms of analgesic effect and safety, the value of clonidine and morphine, used as adjuvants to local anaesthetic, in patient-controlled epidural analgesia (PCEA) after abdominal hysterectomy with midline laparotomy.

\section{Materials and methods:}

Randomized, double-blind, prospective study
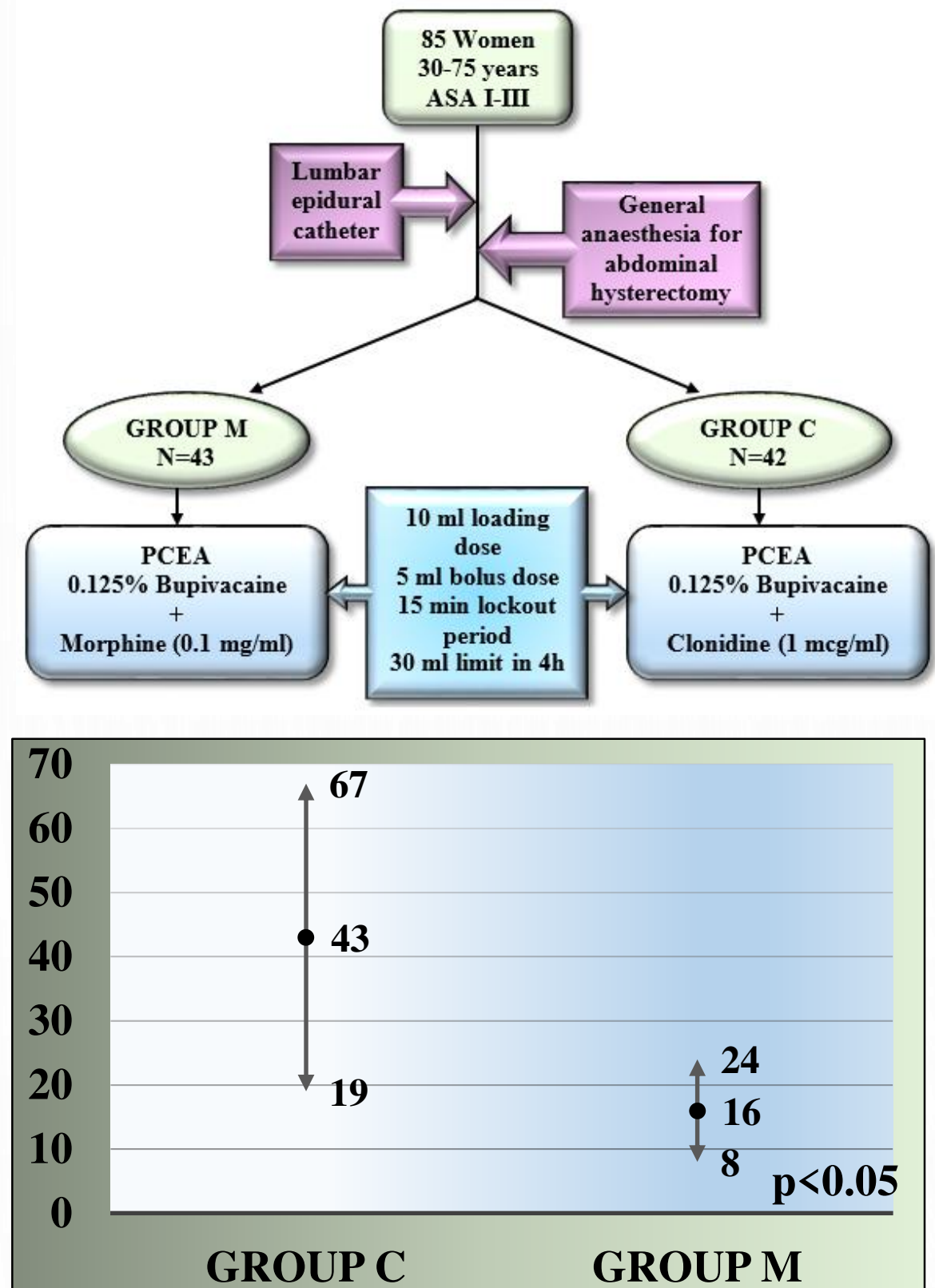

Fig. 3: Time to first bolus request in our groups Time to first bolus request was found significantly longer in group $\mathrm{C}$ versus group $\mathrm{M}$.

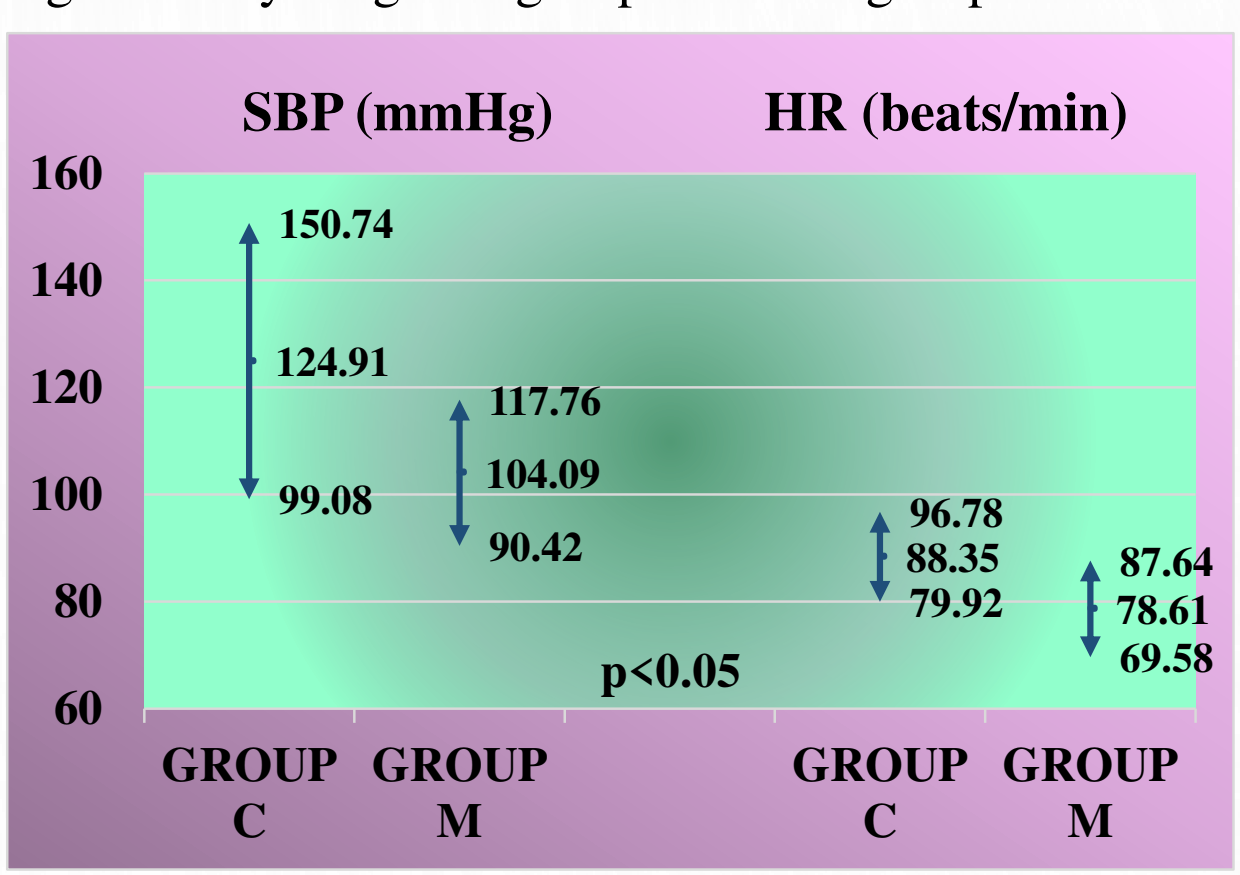

Fig. 6: Hemodynamic status

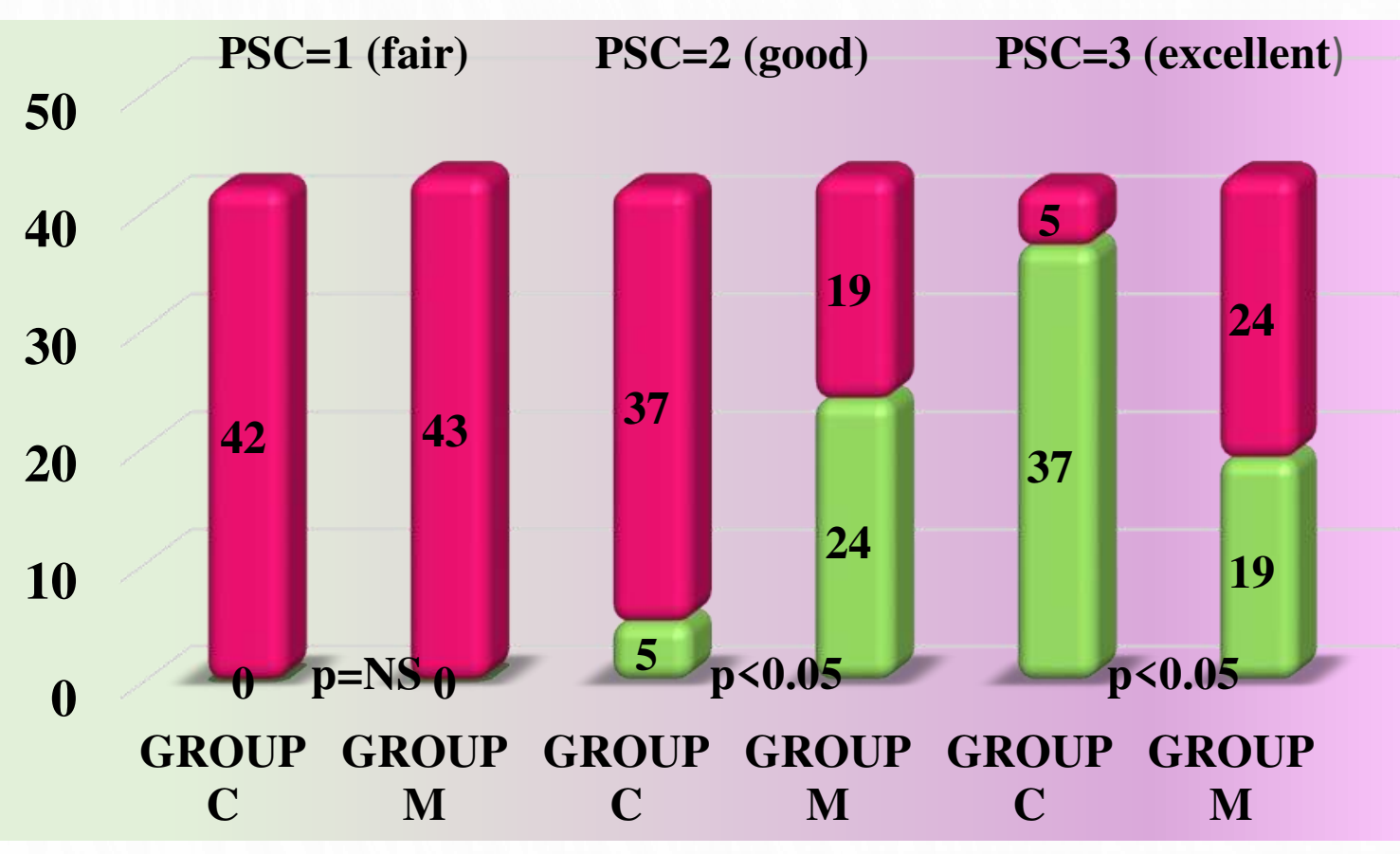

Fig. 9: Patient satisfaction with analgesic regimen

\section{Conclusions:}

Our study concluded that, when administered through PCEA, a solution of $0.125 \%$ bupivacaine plus clonidine 1 meg/ml provided optimal pain control without associated clinically significant morbidity, thus being a better option than combination $0.125 \%$ bupivacaine - morphine $0.1 \mathrm{mg} / \mathrm{ml}$ for acute postoperative analgesia in abdominal hysterectomy patients.

\section{Selective bibliography:}

1. Comparison of effect of epidural bupivacaine, epidural bupivacaine plus fentanyl and epidural bupivacaine plus clonidine on postoperative analgesia afte hip surgery. Kamawat R et al., J. Anesth. Clin. Res. 2013, 4:12.

2. Role of clonidine as adjuvant to intrathecal bupivacaine in patients undergoing lower abdominal surgery: a randomized control study. RB Singh et al Anesth. Essays Res. 2014 sep-dec; 8(3): 307-312.
Primary outcomes: the quality of pain control (VAS) at rest and after coughing, assessed 6 hourly Secondary outcomes: hemodynamic status; incidence of sedation and nausea/vomiting; patient satisfaction (assessed by a four point scale: $0=$ poor, $1=$ fair, $2=$ good, $3=$ excellent).
Results and Discussions:

Demographic variables did not differ significantly among the two groups. None of the patients were excluded from the study.

acute postoperative pain was significantly better controlled, both at rest and after coughing, in group $\mathrm{C}$ compared to group Clonidine, an $\alpha 2$-adrenergic agonist, to bupivacaine solution via PCEA, has synergistic effect on local anaesthetic.

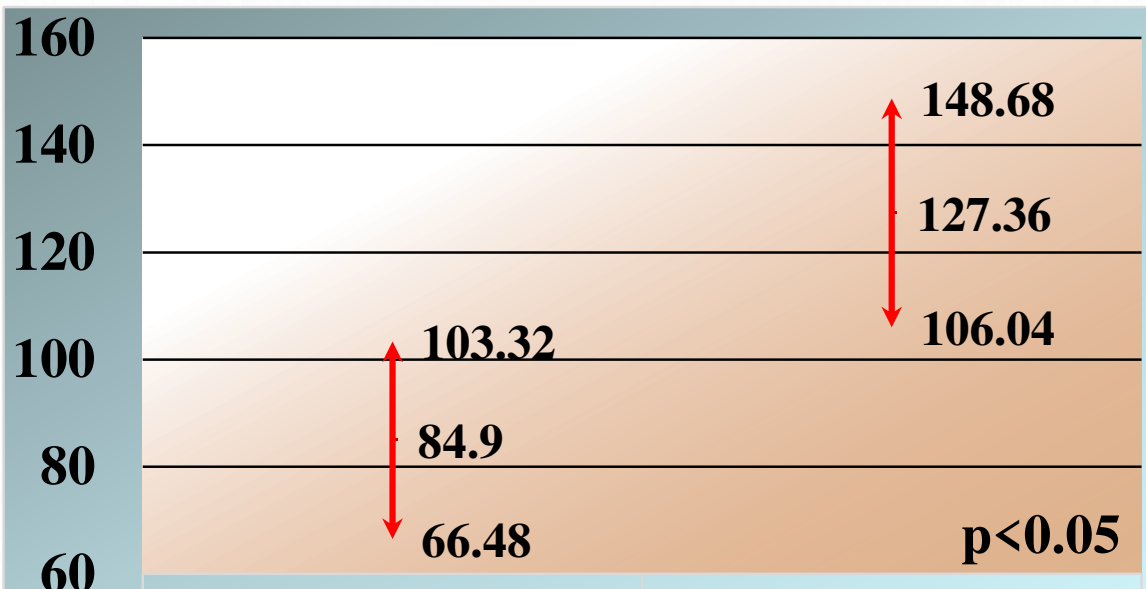

GROUP C

GROUP M

ig. 4: Total analgesic consumption via PCEA

\begin{tabular}{|l|l|l|l|l|}
\hline Group & VAS at 6h & VAS at 12h & VAS at 18h & VAS at 24h \\
\hline Group C & $0.88+/-0.09$ & $1.08+/-0.56$ & $1.15+/-0.94$ & $1.33+/-0.74$ \\
\hline Group M & $1.38+/-0.49$ & $2.04+/-0.79$ & $2.42+/-0.56$ & $2.67+/-0.82$ \\
\hline p & $<0.05$ & $<0.05$ & $<0.05$ & $<0.05$ \\
\hline \multicolumn{5}{|c|}{ Fig. 1: VAS at rest during study period } \\
\hline Group & VAS at 6h & VAS at 12h & VAS at 18h & VAS at 24h \\
\hline Group C & $1.38+/-0.76$ & $1.94+/-0.58$ & $2.11+/-0.57$ & $2.27+/-0.67$ \\
\hline Group M & $2.04+/-0.62$ & $2.72+/-0.60$ & $3.00+/-0.69$ & $3.46+/-0.49$ \\
\hline p & $<0.05$ & $<0.05$ & $<0.05$ & $<0.05$ \\
\hline
\end{tabular}

Fig. 2: VAS after coughing during study period

The interpretation of our results in conjunction with these observations could explain the advantages of clonidine postoperative analgesia in abdominal hysterectomy women. riod revealed that group $\mathrm{C}$ requested lower PCEA analgesic consumption.

Thus, our results demonstrated that clonidine was more potent than morphine when added to bupivacaine via PCEA, in increasing duration of postoperative analgesia and reducing cumulative volume of analgesic solution as well as the need for systemic rescue analgesic. decrease in systolic blood pressure was less than $15 \%$ of baseline during study period.

Concerning sedation, this adverse effect appeared more prominent in group $\mathrm{M}$ compared to group $\mathrm{C}$. However, sedation was mild in all cases and did not Although nausea/vomiting remains a common side effect of postoperative analgesia, patients in group $\mathrm{C}$ experienced lower incidence of this event versus those of group $\mathrm{M}$. The low dose of clonidine added to bupivacaine and delivered by PCEA could explain events, favorable to group $\mathrm{C}$.

Overall satisfaction concerning pain management regimen was good to excellent in both groups, but 37 patients in group C, respectively 19 patients in group $\mathrm{M}$ were fully satisfied by the degree of their pain relief.

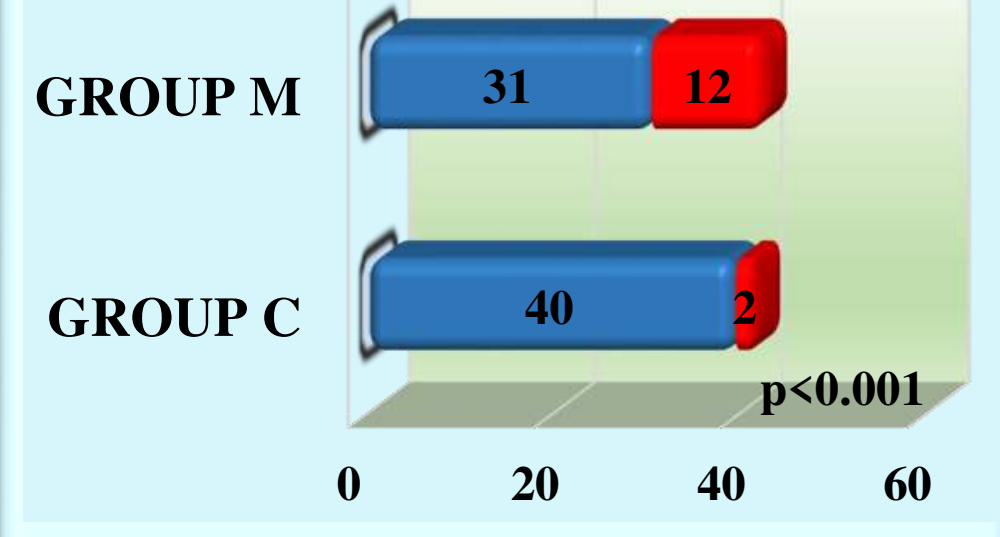

Fig. 7: Incidence of sedation

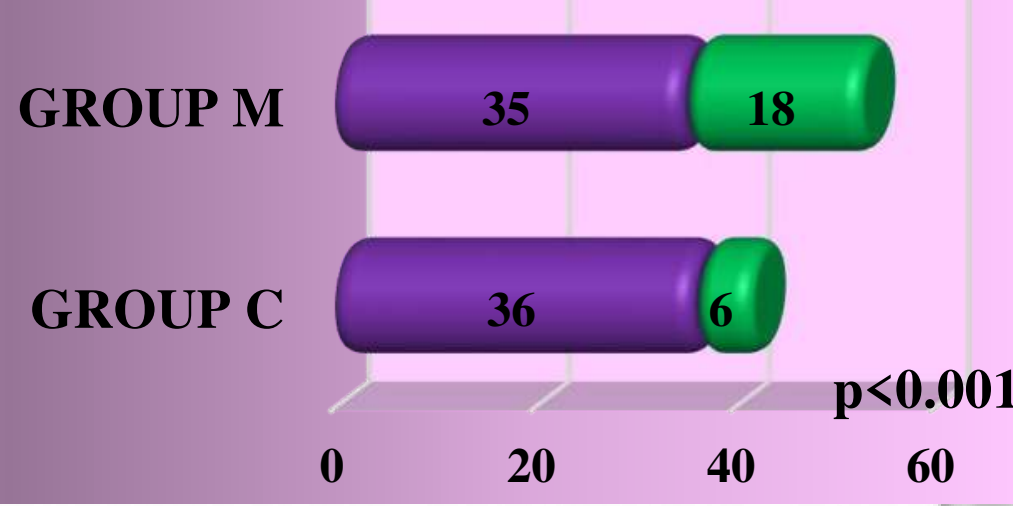

Fig. 8: Incidence of nausea/vomiting statistically significant. However, the fall in both hemodynamic parameters was not clinically such a distribution of side undesirable 